\title{
Contamination of Unused, Nonsterile Gloves in the Critical Care Setting: A Comparison of Bacterial Glove Contamination in Medical, Surgical and Burn Intensive Care Units
}

\author{
Matthew Hall BA, Urvish Trivedi BS, Kendra Rumbaugh PhD, Sharmila Dissanaike MD
}

\begin{abstract}
Objective: Objective: To assess the rate and burden of bacterial contamination on unused, nonsterile gloves found in glove boxes in three different specialty intensive care units (ICUs).

Design: Descriptive, cross-sectional study

Setting: A burn, trauma/surgical, and medical ICU in a 412-bed tertiary care hospital.

Subjects: Convenience sample of 90 non-sterile vinyl exam glove pairs

Methods: Thirty occupied rooms in each ICU were utilized for collection of glove pair samples. Gloves from opened glove boxes placed in wall-mounted racks for use by healthcare staff were donned by one investigator in a routine, aseptic fashion. The surfaces of both gloves were swabbed, plated onto a contact agar plate and incubated for 48 hours. Resulting colony forming units (CFUs) were counted and recorded for each glove pair sample.

Results: Bacterial contaminants were cultured from 73 of 90 (81.1\%) glove pairs sampled across all ICUs. Contamination rates of glove samples from the BICU, SICU and MICU were $66.7 \%, 86.7 \%$ and $90.0 \%$ respectively. The differences in contamination rate among units was statistically significant $(p=0.044)$. The average contamination burden was $5.83 \mathrm{CFU}$ per glove pair and was not significantly different among units.

Conclusions: Despite differences in infection control practices and the composition of pathologies managed in each ICU, the average bioburden of gloves left exposed in the environment was not significantly different. Further research is needed to assess for an association of glove bioburden with nosocomial infection rates and the effects of different infection control practices on the reduction of glove bioburdens.
\end{abstract}

Key words: contamination, ICU, Staphylococci, methicillin resistance

Corresponding author: Matthew Hall BA Contact Information: Matthalltx@gmail.com DOI: $10.12746 /$ swrccc2014.0205.053

\section{INTRODUCTION}

Nosocomial infections are a significant cause of morbidity and mortality in hospitals nationwide and 
are associated with increased lengths of stay, healthcare costs, and resource utilization. ${ }^{1}$ The incidence of nosocomial infections is highest for patients in critical care units (ICUs) with estimates as high as $40 \%$ of ICU admissions; over one-third of all nosocomial infections are acquired in ICUs. ${ }^{2}$

Given the high incidence and burden of these infections, increasing emphasis is being placed on the importance of strict adherence to universal precautions. ${ }^{3,4}$ Regular handwashing, a cornerstone of current universal precautions, is the most effective measure for preventing transmission of infectious organisms in a healthcare environment. ${ }^{5}$ Despite these efforts, poor compliance with adequate handwashing techniques among healthcare workers has remained a substantial barrier to decreasing the rate of nosocomial infections. ${ }^{2,5}$ Use of gloves during patient contact with non-intact skin has become standard practice in the healthcare environment independent of handwashing practice. While routine gloving practices decrease the rate of horizontally transmitted infections, their use without proper handwashing techniques has failed to decrease the rate of nosocomial infections. ${ }^{6}$ This fact has been attributed to inadequate handwashing techniques used prior to donning gloves ${ }^{7}$ and the presence of small undetected holes in the glove material that may provide a conduit to transmit hand flora directly to the patient's skin. It is unknown which environmental factors contribute to the ineffectiveness of gloves in preventing the spread of nosocomial infections.

Previous studies of nonsterile gloves in routine use in the ICU setting have demonstrated a high rate of contamination of unused gloves from opened glove boxes placed in patient care areas, although the overall burden of contamination has been found to be low. ${ }^{7,8}$ Diaz et al. demonstrated that contamination of gloves likely occurred after an opened glove box was exposed to the environment as a result of healthcare workers repeatedly reaching into opened boxes of gloves during the course of the day. To date, no study has compared rates and burden of nonsterile glove contamination among specialty ICUs. Correlation between the composition of glove contamination and organisms found to cause nosocomial infections in the same environment could suggest that contamination of gloves and other areas of the environment have a significant role in the spread of nosocomial infections, independent of hand hygiene practices by healthcare workers. We evaluated glove contaminants by ICU type and the duration of room occupancy at the time of culture.

\section{Materials and Methods}

\section{SETTING}

This study was conducted at University Medical Center, a 412-bed tertiary care hospital in Lubbock, TX, hosting a level 1 trauma center and regional burn center. Thirty glove pair samples were taken from occupied rooms in each of three specialty ICUs at the facility: a 32-bed Medical ICU, a 21-bed Trauma/Surgical ICU, and a 6-bed Burn ICU. Our convenience sample of 90 glove pairs was collected with both isolation status and length of room occupancy recorded at the time of each sampling. All gloves sampled in this study were CURAD ${ }^{\circledR}$ Stretch Vinyl Exam Gloves (Medline CUR9224, CUR9225, CUR9226, CUR9227; Mundelein, IL). In each patient room, access to the glove boxes was unrestricted for all health care providers. The standard aseptic policy in the units is to wash both hands with an antiseptic soap or use an alcohol based solution prior to donning and after discarding boxed, clean, non-sterile gloves. Boxes are routinely designated for each bedside in a dispenser affixed to an adjacent wall. Rooms with patients harboring a resistant pathogen - primarily methicillin resistant Staphylococcus aureus, multi-drug resistant Pseudomonas aeruginosa, or Clostridium difficile were designated isolation rooms per hospital policy, as were all rooms within the Burn ICU. These rooms had a separate cart containing paper gowns, caps, and masks in front of each room for use before entering the room in addition to the standard glove boxes. Permission to conduct the study was obtained from both physician and nursing supervisors in each unit.

\section{Sample Collection}

Two researchers (MH, UT) performed all sampling of gloves. Attending health care personnel were given no prior notification of the sampling times which 
occurred at random dates and times. The nature and intent of the study were not disclosed to unit staff in order to minimize deviation from standard routine. Standard aseptic hand washing was done before the investigator donned a new pair of sterile gloves chosen from one of the boxes in each room. Then each fingertip, digit, and palm of both hands were swabbed using moistened six inch sterile cotton tipped applicators (Fisher Scientific, Waltham, MA) which were lightly rolled over the surface of sterile contact plates containing tryptic soy agar with lecithin and polysorbate 80 (Remel, Lenexa, KS). This method was repeatedly employed for all the sampled bedside rooms. The unit and bedside number were recorded and samples were given ID numbers. A total of 90 samples were employed, 30 samples from three different units, for this study.

\section{MiCROBIOLOGY}

The contact plates were incubated at $37^{\circ} \mathrm{C}$ and inspected after 48 hours, and the number of colonyforming units (CFU) per sample was recorded. Each colony was given a unique ID number corresponding with the bedside rooms sample IDs, enabling the isolation and differentiation of different organisms within each study sample. Each isolated, morphologically distinct representative colony on the contact plates was then plated onto Luria-Bertani agar (Difco Laboratories, Becton, Dickinson and Company, Sparks, MD) and Gram-negative selective MacConkey agar (Remel, Lexena, KS) in $100 \mathrm{~mm} \times 15 \mathrm{~mm}$ polystyrene Petri dishes using individually wrapped sterile inoculating loops (Fisher Scientific, Waltham, MA). Samples were incubated at $37^{\circ} \mathrm{C}$ for 48 hours, at which point any bacterial colonies grown on the agar were examined and recorded as positive or negative growth. Colonies that grew on MacConkey agar were plated on selective Pseudomonas Isolation agar (Difco Laboratories, Becton, Dickinson and Company, Sparks, MD) for positive screening and identification of Pseudomonas. Colonies which grew only on Luria-Bertani agar were further screened using Staphylococcus medium 110 agar (Difco Laboratories, Becton, Dickinson and Company, Sparks, MD) for isolating and differentiating Staphylococci and Mueller Hinton agar with $4 \% \mathrm{NaCl}$ and $6 \mu \mathrm{g} / \mathrm{mL}$ oxacillin (Remel, Lenexa, KS) for screening and iso- lating methicillin-resistant Gram-positive organisms. (Oxacillin-resistant colonies are henceforth referred to as methicillin-resistant in this paper) Colonies were incubated at $37^{\circ} \mathrm{C}$, inspected, and counted after 48 hours of incubation and recorded as positive or negative for growth. Contaminated gloves were defined as those from which any detectable bacteria grew on contact plates following a 48-hour incubation period.

\section{Data analysis}

Contamination rate analysis was conducted using a Chi-square test for proportions to measure the significance of the differences in glove pair contamination rates between specialty ICUs and between samples grouped based on the isolation status of the room from which they were obtained. A Chi-square test for proportions was also used to compare differences in the rate of methicillin-resistant organism contamination on glove pairs grouped by specialty ICU and by room isolation status. Kruskal-Wallis tests were performed to analyze differences in the average contamination burden of glove pair samples between specialty ICUs. Kruskal-Wallis tests were also performed to assess the differences in the mean number of distinct morphologic subtypes per glove pair sample among specialty ICUs. A Mann-Whitney U-test was performed to assess for statistically significant differences among samples gathered from isolation and non-isolation status rooms in total contamination burden per sample and average number of distinct morphologic subtypes per sample. Finally, linear regression plots were constructed to attain a coefficient of determination to assess the strength of the correlation between total contamination burden and duration of room occupancy at time of sampling. All analyses were performed using Microsoft Excel 2010 (Microsoft Corporation, Redmond, WA).

\section{RESULTS}

We found an average contamination rate of $81.1 \%$ across all glove pairs sampled with an average bioburden of 5.83 CFU (SD = 8.04). Our findings are consistent with previously reported rates of several studies describing contamination rates of $55-87 \%$ and average bioburdens ranging from 3.4-6.2 CFU per glove pair. ${ }^{7-9}$ Significant differences in the rate of 


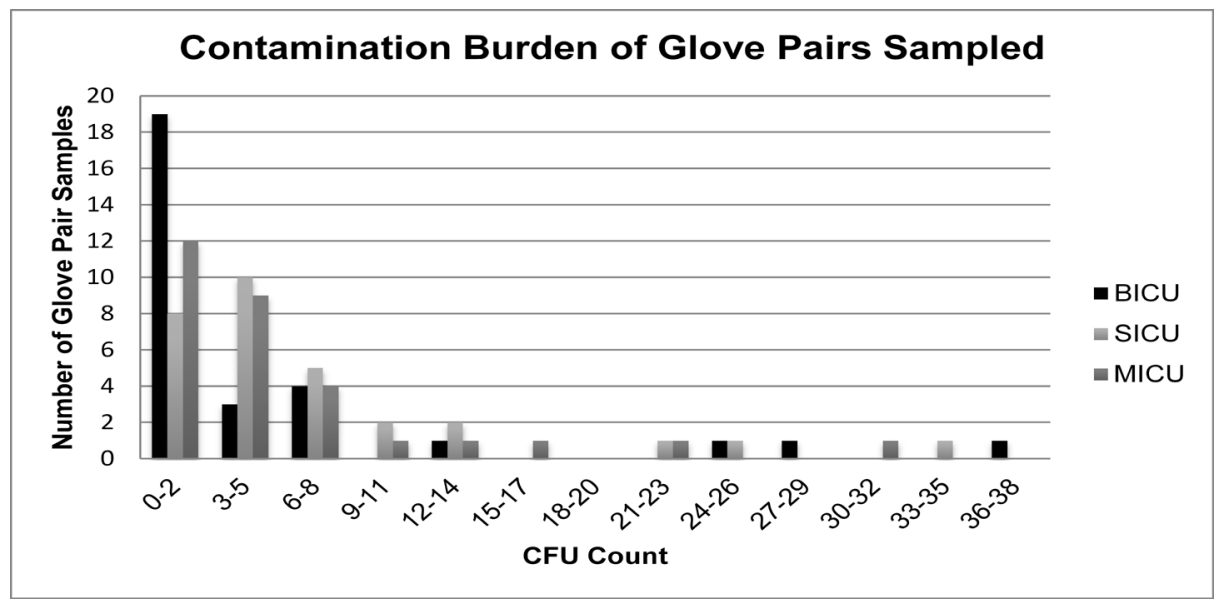

Figure 1: Contamination Burden of Glove Pairs Sampled. Histogram demonstrating the number of samples for each range of colony forming units cultured onto initial contact plate.

Table 1: Contamination Rate of Glove Samples

\begin{tabular}{|l|c|c|}
\hline Sample: & Contaminated Samples (\%): & MRO-Contaminated Samples (\%): \\
\hline BICU: & $20(66.7 \%)$ & $9(25.7 \%)$ \\
\hline SICU: & $26(86.7 \%)$ & $14(46.7 \%)$ \\
\hline MICU: & $27(90.0 \%)$ & $10(33.3 \%)$ \\
\hline \multicolumn{3}{|c|}{} \\
\hline Isolation: & $27(71.1 \%)$ & $12(31.6 \%)$ \\
\hline Non-Isolation: & $46(88.5 \%)$ & $21(20.0 \%)$ \\
\hline \multicolumn{3}{|c|}{} \\
\hline All Samples: & $73(81.1 \%)$ & $33(36.7 \%)$ \\
\hline
\end{tabular}

Table 2: Average Contamination Burden of Glove Pair Samples

\begin{tabular}{|c|c|c|c|c|c|c|c|c|}
\hline \multirow[t]{2}{*}{ Sample: } & \multirow{2}{*}{$\begin{array}{l}\text { Sample } \\
\text { Size (N): }\end{array}$} & \multirow[t]{2}{*}{ Mean: } & \multirow{2}{*}{$\begin{array}{l}\text { Standard } \\
\text { Deviation: }\end{array}$} & \multirow[t]{2}{*}{$95 \% \mathrm{Cl}:$} & \multicolumn{2}{|c|}{$95 \% \mathrm{Cl}$} & \multirow[t]{2}{*}{ Min: } & \multirow[t]{2}{*}{ Max: } \\
\hline & & & & & $\begin{array}{l}\text { Lower } \\
\text { Bound: }\end{array}$ & $\begin{array}{l}\text { Upper } \\
\text { Bound: }\end{array}$ & & \\
\hline BICU: & 30 & 5.2 & 9.32 & 3.48 & 1.72 & 8.68 & 0 & 38 \\
\hline SICU: & 30 & 6.97 & 7.96 & 2.97 & 4.00 & 9.94 & 0 & 35 \\
\hline MICU: & 30 & 5.33 & 6.79 & 2.54 & 2.80 & 7.87 & 0 & 31 \\
\hline Isolation: & 38 & 5.26 & 8.60 & 2.83 & 2.44 & 8.09 & 0 & 38 \\
\hline $\begin{array}{l}\text { No Isola- } \\
\text { tion: }\end{array}$ & 52 & 6.25 & 7.66 & 2.13 & 4.12 & 8.38 & 0 & 35 \\
\hline $\begin{array}{l}\text { All Sam- } \\
\text { ples: }\end{array}$ & 90 & 5.83 & 8.04 & 1.68 & 4.15 & 7.52 & 0 & 38 \\
\hline
\end{tabular}

Identification of a representative from each morphologic subtype isolated was performed using the selective culturing methods described in the Methods are shown in Table 3. No Gram-negative bacteria were isolated throughout the culturing process across all samples. Methicillin-resistant organism (MRO) contamination of glove pairs was prevalent in all units, with more than one in three glove pairs sampled demonstrating contamination with methicillin-resistant organisms. However, rates of MRO contamination of glove samples were not significantly different among the burn, trauma/surgical, and medical ICUs $(p=0.35)$. 
Table 3: Identification of Morphologically Distinct Colonies Isolated:

\begin{tabular}{|l|l|l|l|l|l|l|}
\hline Subtype ID: & BICU: & SICU: & MICU: & $\begin{array}{l}\text { Isolation } \\
\text { Rooms: }\end{array}$ & $\begin{array}{l}\text { Non- } \\
\text { Isolation } \\
\text { Rooms: }\end{array}$ & $\begin{array}{l}\text { All Sam- } \\
\text { ples: }\end{array}$ \\
\hline MSS (\%): & $22(62.9 \%)$ & $45(70.3 \%)$ & $41(74.6 \%)$ & $32(65.3 \%)$ & $76(72.4 \%)$ & $108(70.1 \%)$ \\
\hline MRS (\%): & $7(20.0 \%)$ & $13(20.3 \%)$ & $9(16.4 \%)$ & $9(18.4 \%)$ & $20(19.1 \%)$ & $29(18.8 \%)$ \\
\hline MRNSGP (\%): & $2(5.7 \%)$ & $3(4.7 \%)$ & $1(1.8 \%)$ & $3(6.1 \%)$ & $1(1.0 \%)$ & $3(2.0 \%)$ \\
\hline MSNSGP (\%): & $1(2.9 \%)$ & $3(4.7 \%)$ & $1(1.8 \%)$ & $2(4.1 \%)$ & $5(4.8 \%)$ & $8(5.2 \%)$ \\
\hline Unknown (\%): & $3(8.6 \%)$ & $0(0.0 \%)$ & $3(5.6 \%)$ & $3(6.1 \%)$ & $3(2.9 \%)$ & $6(3.9 \%)$ \\
\hline Total: & 35 & 64 & 55 & 49 & 105 & 154 \\
\hline
\end{tabular}

MSS: methicillin-sensitive Staphylococci; MRS: methicillin-resistant Staphylococci; MRNSGP: methicillin-resistant, non-Staphylococcus Gram-positive; MSNSGP: methicillin-sensitive, non-Staphylococcus Gram positive

Finally, we assessed the correlation of contamination burden of each glove sample with the length of time that the room from which the sample was obtained had been occupied at the time of sampling. These data were analyzed with a linear regression plot (Figure 2) and failed to demonstrate a significant correlation between duration of room occupancy and total contamination burden for all samples $(R 2=0.0113, p=0.318)$ or for samples grouped by ICU type (BICU: R2 $=0.0716, p=0.153$; SICU: R2 $=0.0005, p=0.910 ;$ MICU: R2 = $0.0364, p=0.312$ ).

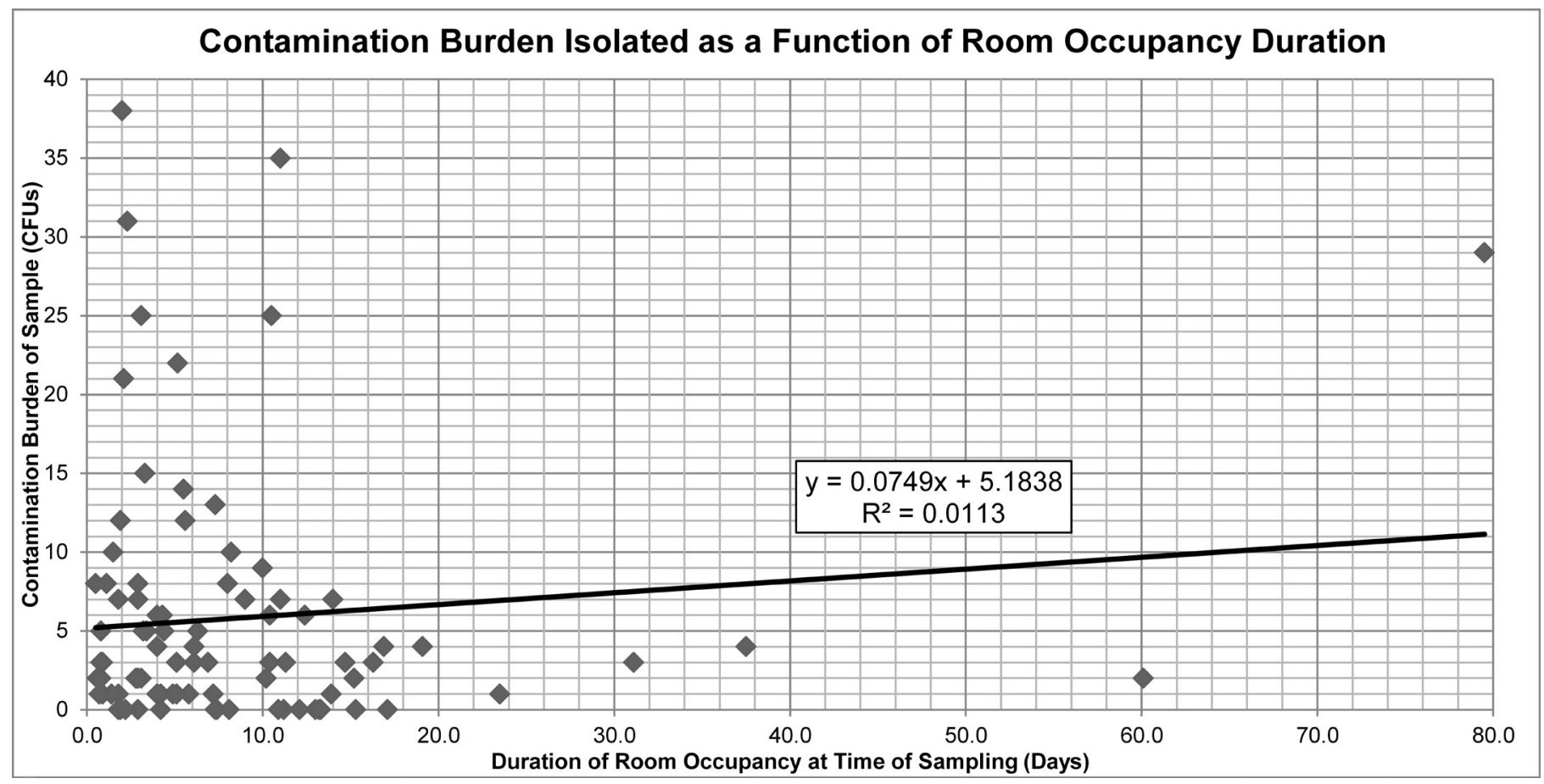

Figure 2: Contamination Burden as a Function of Room Occupancy Duration. 
sample contamination were found between the three ICUs, and differences in average contamination burden per sample (Figure 1, Table 2) approached statistical significance $(p=0.051)$. All BICU rooms, 5 SICU rooms, and $3 \mathrm{MICU}$ rooms had isolation precautions in effect at the time of sampling. Data grouped by room isolation status (Table 1 ) showed a statistically significant difference in the rate of glove contamination ( $p=0.037$ ), but differences in average contamination burden of samples grouped by room isolation status were not statistically significant.

Identification of a representative from each morphologic subtype isolated was performed using the selective culturing methods described in the Methods are shown in Table 3. No Gram-negative bacteria were isolated throughout the culturing process across all samples. Methicillin-resistant organism (MRO) contamination of glove pairs was prevalent in all units, with more than one in three glove pairs sampled demonstrating contamination with methicillin-resistant organisms. However, rates of MRO contamination of glove samples were not significantly different among the burn, trauma/surgical, and medical ICUs $(p=0.35)$.

Finally, we assessed the correlation of contamination burden of each glove sample with the length of time that the room from which the sample was obtained had been occupied at the time of sampling. These data were analyzed with a linear regression plot (Figure 2) and failed to demonstrate a significant correlation between duration of room occupancy and total contamination burden for all samples (R2 = $0.0113, p=0.318$ ) or for samples grouped by ICU type (BICU: R2 = 0.0716, $p=0.153 ;$ SICU: R2 $=0.0005, p$ $=0.910 ; \mathrm{MICU}: \mathrm{R} 2=0.0364, \mathrm{p}=0.312$ ).

\section{Discussion}

Despite differences in infection control practices and the composition of pathologies managed in each specialty ICU, the average bioburden of gloves left exposed in the environment was not significantly different between ICUs. Significant differences do exist, however, in the rates of contamination of glove pair samples when grouped by ICU type. These differences may be attributable to differences in infec- tion control practices both at the environmental and healthcare provider level. Specifically, the lower contamination rate observed in the BICU may be the result of strict contact precautions utilized for all occupied rooms in this unit, regardless of the infectious status of the patient due to the unique vulnerability of the burned patient to infection. This includes replacing glove boxes in each room every time a patient is discharged from the room, likely resulting in a much higher turnover of fresh gloves than is seen in the MICU or SICU, where this practice was followed only if patients are placed in isolation precautions due to suspected or confirmed communicable infection. Furthermore, rooms in the BICU undergo routine ultraviolet radiation antimicrobial treatment, likely resulting in a lower environmental contamination burden by eliminating reservoirs of contamination that may play a part in cross-contamination of unused gloves in the environment. The contamination rates of samples taken from the SICU and MICU, which follow similar and less-stringent infection control protocols than the BICU, differ only by one sample, further supporting this theory.

The failure to isolate a single Gram-negative organism from any of the gloves sampled suggests that unused gloves exposed to the critical care environment do not serve as a reservoir of pathogenic bacteria. This finding is especially significant in the case of the BICU from which samples were obtained, as several multidrug-resistant Pseudomonas infections had been reported in the unit over the time period of the study. However, significant differences in the rates of potentially pathogenic bacteria on unused gloves may reflect differences in the prevalence of these bacteria in each environment.

This study also assessed whether gloves obtained from rooms with different durations of occupancy revealed different levels of contamination. Previous studies have failed to find a correlation between the time that opened boxes of gloves were left exposed in the ICU environment and the contamination burden found on exposed gloves.8 We sought to examine whether the time interval between the more through environmental cleaning procedures performed in empty rooms between patient occupancy periods had any effect on bacterial burden found on gloves. The 
data failed to reveal a correlation between the duration of time the room from which a sample was obtained was occupied and the contamination burden found on the sample. Although we were unable to control for how long each specific glove box from which samples were obtained had been present in the environment, our findings suggest that unused glove contamination burden is not dependent on room occupancy duration.

Lastly, we determined the percentage of morphologically distinct colonies that displayed methicillin-resistance during the selective culturing identification process outlined above. We found that $23 \%$ of the colonies that underwent the subculture identification process showed methicillin resistance. Because only morphologically distinct bacterial colonies were subcultured, we are unable to report an accurate rate of contamination of glove pairs with MRSA. However, we did find evidence of MRO contamination on $36.7 \%$ of glove pairs sampled. Because our contamination rate estimate most likely underestimates the true rate of MRO contamination due to the selective culturing process followed, actual rates of MRO contamination may be higher than those reported in this study. The significance of our finding is difficult to determine without more accurate data on contamination rates and the burden of MRO organisms, and more study is warranted to assess whether MRO contamination of unused nonsterile gloves is high enough to be considered a reservoir source of these pathogenic bacteria.

While our study failed to demonstrate a significant difference in contamination burden among three specialty ICU settings despite differences in infection control practices, our results indicated a trend toward lower contamination burden with the use of conventional contact precautions. More research is needed to assess for an association of glove bioburden with nosocomial infection patterns. Should evidence suggesting a relationship be found, the effects of different infection control practices on the reduction of glove bioburden should be more thoroughly investigated to identify methods for optimizing these practices as a potential means of reducing contamination.

Our study has several important limitations. First, the methods we employed for identifying con- taminants were not specific enough to determine which species of Staphylococci were isolated. While our study does elucidate what percentage of staphylococcal contaminants was methicillin-resistant, we are unable to definitively identify what proportion of these contaminants was $S$ aureus. The distinction is needed to further assess the percentage of contaminants that are known to be virulent, since other, more benign Staphylococcal species may have acquired drug resistance. Additional studies using more specific identification techniques, such as polymerase chain reaction, are needed. Secondly, our study does not compare contamination rates of gloves from unopened glove boxes. It is possible that some contaminants were introduced onto the gloves during the manufacturing and packaging process and are not due to environmental contamination in the ICU. More studies are needed to assess when these contaminants are introduced onto the gloves and also whether contamination increases with the length of time an open box is left exposed in the ICU environment.

KEY WORDS- contamination, ICU, Staphylococci, methicillin resistance

Author Affiliation: Matthew Hall and Urvish Trivedi are medical students in the School of Medicine at TTHUSC. Kendra Rumbaugh and Sharmila Dissanaike are faculty members in the Department of Surgery in the School of Medicine at TTUHSC..

Received: $10 / 10 / 2013$

Accepted: $12 / 10 / 2013$

Reviewers: Vipul Desai MD, Mongkolrattanothai, Kanokporn MD

Published electronically: 1/15/2014

Conflict of Interest Disclosures: None 


\section{REFERENCES}

1. Vincent JL. Nosocomial infections in adult intensive-care units. Lancet 2003; 361(9374):2068-77.

2. Eggimann P, Pittet D. Infection control in the ICU. Chest 2001; 120(6):2059-93.

3. Tschudin-Sutter S, Pargger H, Widmer AF. Hand hygiene in the intensive care unit. Crit Care Med 2010; 38(8 Suppl):S299-305.

4. Patterson JE, Malani PN, Maragakis LL. Infection control in the intensive care unit: progress and challenges in systems and accountability. Crit Care Med 2010; 38(8 Suppl):S265-268.

5. Centers for Disease Control and Prevention. Public health focus: surveillance, prevention, and control of nosocomial infections. MMWR Morb Mortal Wkly Rep 1992; 41:783787.

6. Olsen RJ, Lynch P, Coyle MB, Cummings J, Bokete, T, Stamm, W. Examination gloves as barriers to hand contamination in clinical practice. JAMA 1993; 270(3): 350-353.

7. Diaz MH, Silkaitis C, Malczynski M, Noskin G, Warren $\mathrm{J}$, Zembower T. Contamination of examination gloves in patient rooms and implications for transmission of antimicrobial-resistant microorganisms. Infect Control Hosp Epidemiol 2008; 29(1):63-65.

8. Rossoff LJ, Lam S, Hilton E, Borenstein M, Isenberg H. Is the use of boxed gloves in an intensive care unit safe? Am J Med 1993; 94(6)602-607.

9. Ferreira AM, Andrade DD, Haas VJ. Microbial contamination of procedure gloves after opening the container and during exposure in the environment. Rev Esc Enferm USP 2011; 45(3): 745-750. 